\title{
High finesse compound optical ring filter for parametric multicasting RF channelization
}

\author{
J. R.Adleman, S. Zlatanovic, J.M. Kvavle, B.M.L. Pascoguin, E.W. Jacobs \\ Advanced Photonics Technologies Branch \\ SPAWAR Systems Center Pacific \\ San Diego, CA, USA \\ james.adleman@navy.mil
}

\begin{abstract}
An integrated optical compound ring filter for use in a multicast and slice channelizer/spectrum analyzer is presented. We discuss design and implementation of a $\mathrm{SiO}_{2}$ based photonic filter with $250 \mathrm{MHz}$ resolution bandwidth, a flattened passband, and finesse greater than 100, enabling channelization of instantaneous bandwidths above $10 \mathrm{GHz}$.
\end{abstract}

Keywords-microwave photonics; photonic integrated circuits; resonators; (key words)

\section{INTRODUCTION}

\section{A. Motivation}

The many $\mathrm{THz}$ of useful bandwidth available in optical waveguides positions RF photonics as a vital technique for radio communication and surveillance for wideband (tens of gigahertz) signals [1]. The implementation of a $100 \%$ duty cycle (i.e. staring mode) real time RF spectral analyzer and/or channelizer has been previously pursued using banks of narrowly spaced, high quality electrical or optical domain RF filters with 1 filter per channel. This approach is limited by the need to carefully align multiple filter center frequencies and minimize crosstalk between channels. Discrete filters arrays tend to be expensive both in cost and in size, weight, and power (SWaP) considerations.

High resolution channelization of wideband signals with reduced SWaP becomes feasible if the constraints of tight spacing and alignment of individual filters can be eliminated. Recently the use of parametric multicasting to create an array of high fidelity copies of an RF photonic signal across the whole optical C-band has been utilized to implement a staring mode spectrum analyzer/channelized receiver [2]. A single, periodic passband optical filter is used to slice a narrow channel out of each copy, creating high resolution bandwidth (RBW) channels spaced by tens of gigahertz in the optical spectrum. The absolute frequency positioning of the passbands is no longer a critical parameter as the copies can easily be aligned to the filter by adjusting the multicaster. However, conventional optical filters such as fiber Fabry-Perot etalons are not ideal for channelization due to large passband slope leading to loss of signal fidelity, and wide transition bandwidths leading to significant crosstalk between channels. These limitations create a new demand for a class of high finesse optical filters that have flattened passbands, rapid transition bandwidths, and narrow passband width. In this work, we present the design and characterization of such a filter, implemented as a photonic integrated circuit (PIC) using multiple stages of optical ring lattices.

\section{DESIGN}

In order to meet channelizer system requirements of RBW $<250 \mathrm{MHz}$ and RF instantaneous bandwidth (IBW) $>10 \mathrm{GHz}$, a minimum filter finesse of 100 was chosen as a design goal. We use ring resonators as the building block for the filter design, as they are strictly periodic in frequency, can be created in a PIC platform with moderate quality factors, and their passband alignment can be easily tuned across the FSR using a thermo-optic phase shift. Ring lattice configurations, where individual rings with identical FSRs are directly coupled to each other via directional couplers, can be used to create flattened periodic passbands with RBWs that are small fraction of the FSR [3]. However, the achievable finesse of a ring lattice constitutes a trade off with the acceptable insertion loss of the filter, and is dictated by the propagation loss of the PIC waveguides. In order to optimize insertion loss and still meet the $\mathrm{RBW}$ goal a low loss $\mathrm{SiO} 2$ on $\mathrm{Si} \mathrm{PIC}$ platform with propagation loss of $2 \mathrm{~dB} / \mathrm{m}$ was chosen.

We implemented an infinite impulse response (IIR) approximation to a Butterworth filter using two rings via the design method outlined in [4], which allows finesse of up to around 10 while maintaining passband flatness better than 1 $\mathrm{dB}$. Therefore the maximum FSR that could be achieved using this two ring lattice stage was approximately $2.5 \mathrm{GHz}$. In order to increase the finesse we cascaded multiple ring lattice stages with different FSRs together. This creates a filter with an effective FSR determined by the least common multiple of the single stage FSRs, in an example of the Vernier effect. A schematic diagram of the architecture is shown in figure 1 . The filter design for each lattice stage is completely specified by determining the power coupling ratios between the rings and the bus waveguides (viz. $\kappa_{1 A}, \kappa_{A B}, \kappa_{B 2}$ ) the phase of each ring (viz. $\varphi_{A}, \varphi_{B}$ ) and the desired FSR for the stage (e.g. $\left.F S R_{A B}\right)$. The $\varphi$ and $\kappa$ values are calculated from the approximate filter's transfer function using a step down recurrence relation [5]. The total length of each individual ring is calculated as:

$$
\mathrm{L}_{\mathrm{A}, \mathrm{B}}=\mathrm{c}_{0} /\left(\mathrm{n}_{\mathrm{eff}} \mathrm{FSR}_{\mathrm{AB}}\right) \text {. }
$$


Since the ring lengths in excess of $10 \mathrm{~cm}$ are needed, individual rings are made more compact on chip by implementing a scroll shape as shown in the Fig.1 inset.

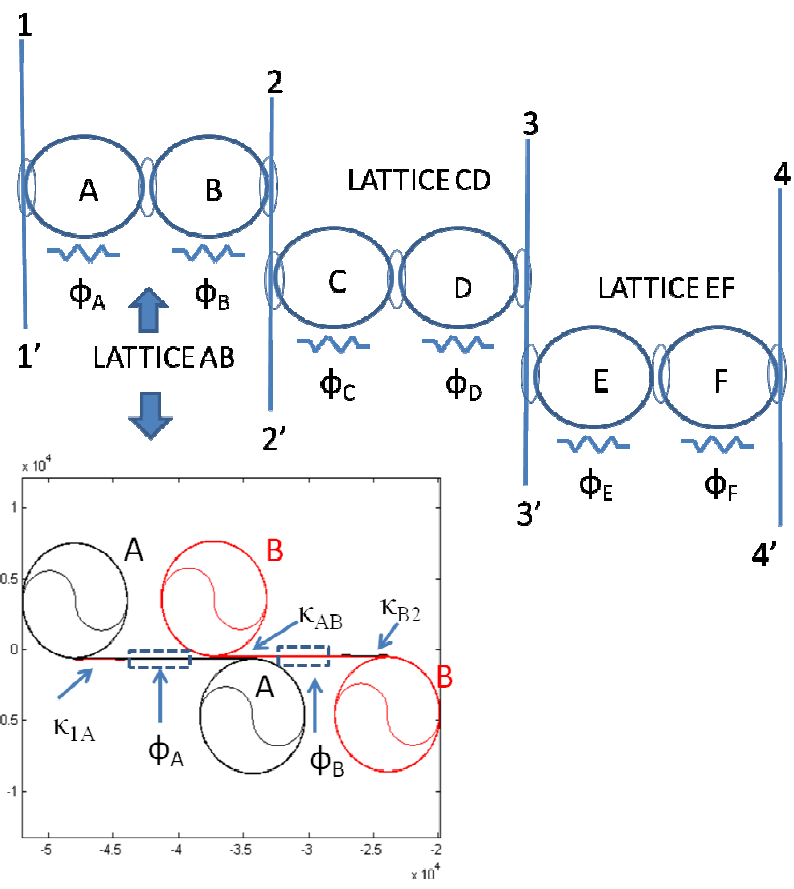

Fig. 1. Schematic diagram of a 3 stage ring lattice filter, where rings are denoted by letters A-F. Stages are connected via bus waveguides 1-4. Shaded ovals represent directional couplers between elements. Inset is a 1.0 $\mathrm{GHz}$ ring lattice stage as laid out on chip, with $\kappa_{1 \mathrm{~A}}=0.571, \kappa_{\mathrm{AB}}=0.188, \kappa_{\mathrm{B} 2}$ $=0.424, \varphi_{\mathrm{A}, \mathrm{B}}=0$; size of scale box is $\sim 2.5 \mathrm{~cm} \mathrm{X} 2.5 \mathrm{~cm}$. Dotted rectangles denote are allowed area for integration of resistive heaters.

Resistive heating regions are defined along the center "spine" of each ring to allow thermo-optic phase shifting to individually tune each ring's phase (passband alignment).

The length of each directional coupling region, $L_{\kappa}$, is determined via:

$$
\kappa=\sin ^{2}\left(k_{e f f} L_{\kappa}\right)
$$

where $k_{\text {eff }}$ is a coupling constant determined via a commercial beam propagation solver (RSoft BeamProp) using the waveguide geometry and spacing between coupler waveguides as input parameters.

Six ring (three stage) PIC filters with effective FSRs of $5 \mathrm{GHz}$, 6.25 GHz, and $10 \mathrm{GHz}$ were designed as shown in Fig. 2. The $6.25 \mathrm{GHz}$ filter can be cascaded with the $5 \mathrm{Ghz}$ filter to produce a $25 \mathrm{Ghz}$ FSR 6 stage filter, or with the $10 \mathrm{GHz}$ filter to produce a $50 \mathrm{GHz} 6$ stage filter. These compound filters are designed for extinction rations of better than $20 \mathrm{~dB}$, and minimum insertion loss below $6 \mathrm{~dB}$.

Sponsored by Office of Naval Research Electronic Warfare Discovery and Invention program and SSC Pacific In House Laboratory Independent Research program

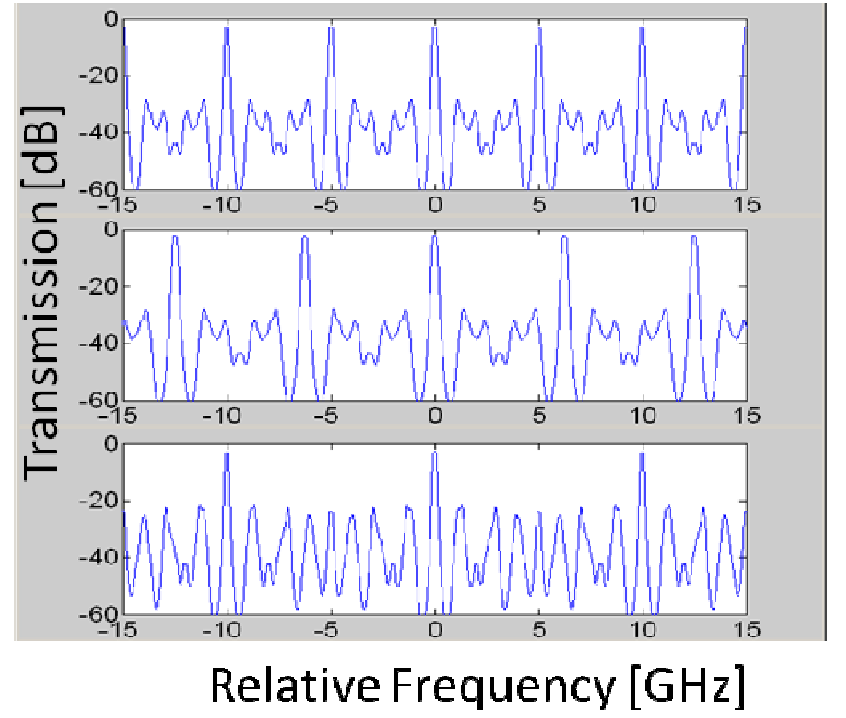

Fig. 2. Designed magnitude response for three stage compound filters: Top effective FSR 5GHz, Middle FSR $6.25 \mathrm{GHz}$, Bottom FSR $10 \mathrm{GHz}$.

\section{RESULTS}

The designed filters were custom fabricated in a low loss $\mathrm{SiO}_{2}$ on $\mathrm{Si}$ PIC platform by Enablence USA. Nominal waveguide dimensions of $4 \times 4$ microns, index contrast of $\Delta=$ $1.5 \%$, and loss of $2 \mathrm{~dB} / \mathrm{m}$ were used as parameters of the design. Resistive heating phase shifters for each ring were fabricated with resistances of approximately $100 \mathrm{ohms}$. Each PIC is packaged with thermoelectric cooling to maintain the overall chip temperature. The devices are pigtailed with a polarization maintaining fiber arrays, and fiber to chip pigtail coupling loss is estimated to be between $1-2 \mathrm{~dB}$.

The magnitude response of the filters was measured with an optical frequency domain reflectometer (Luna OBR 4400), using a circulator to feed light transmitted through the device under test back into the reflectometer. Magnitude response of each individual lattice is obtained by sending light backward (e.g. from port 2 to port 1'to measure Lattice $A B$ in isolation as in Fig.1). Fig.3 shows the magnitude response of a lattice stage before and after phase tuning. Minor imperfections in fabrication create a relative phase shift between the two coupled rings that degrade the passband performance. The phase difference can be compensated by heating one of the two rings. Each phase shifter is driven independently from a shared $10 \mathrm{~V}$ supply using pulse width modulation, with pulses at $10 \mathrm{KHz}$ repetition rate. Even when the lattice stage is optimized, we observe a degraded extinction ratio as compared to the design. This can be attributed to a discrepancy in the both the nominal propagation loss and coupling constants used in the design with those actually fabricated on the PIC.

Although each lattice can be heated independently, noticeable thermal cross talk occurs between neighboring stages which complicates concurrent alignment of the compound filter. In principle the thermal crosstalk can be characterized and calibrated out. However, we can also 
determine the optimal performance of the fabricated device by individually aligning

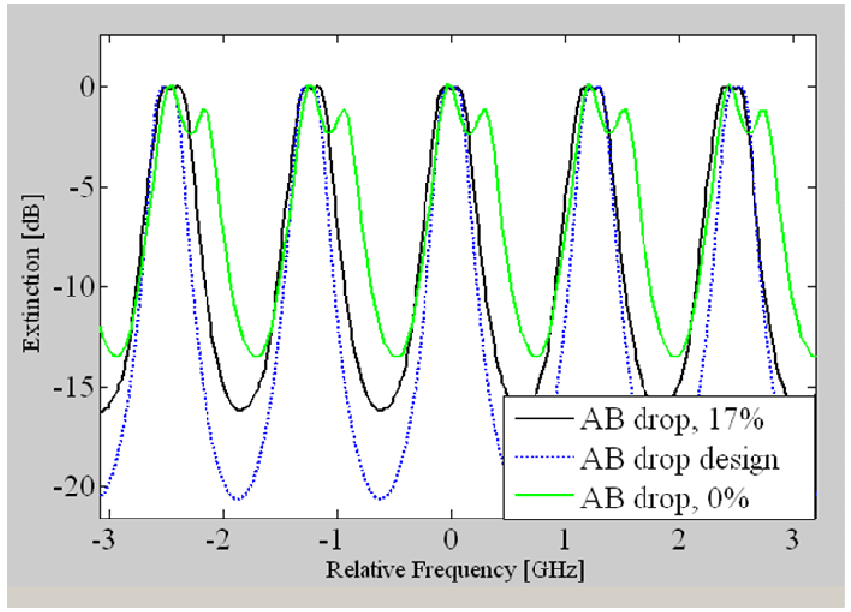

Fig. 3. Measured extinction of a $1.25 \mathrm{GHz}$ FSR ring lattice stage as fabricated ( $0 \%$ heater duty cycle) and tuned (17\% duty cycle) as compared to design. Curves are normalized to peak transmission for each case.

each stage with all other stage heaters turned off, and numerically combining the measured results. Fig 4 shows the resultant magnitude response (normalized extinction) for the fabricated $6.25 \mathrm{GHz}$ FSR 3 stage cascade, and a $25 \mathrm{GHz}$ FSR 6 stage cascade, as compared to the designed magnitude response The overall shape of the measured filter passbands is in good agreement with the design, however, a degradation of the filter extinction ratio from better than $25 \mathrm{~dB}$ to approximately $18 \mathrm{~dB}$ observed. Additionally minimum insertion losses are estimated to be as much as $6 \mathrm{~dB}$ higher than the $6 \mathrm{~dB}$ IL of the design (not shown in Fig.4). We once again attribute this to increased propagation loss and decreased coupling constant, which cause excess energy to be consumed by absorption and scattering with in the ring resonators. We are currently performing a parametric analysis and curve fitting to determine the magnitude of loss and coupling errors from the measured data, which will be reported on presently.

\section{CONCLUSION}

We have developed a PIC compound ring resonator filter composed of cascaded stages of ring lattice elements. A fabricated 6 stage filter has an optimized passband width of approximately $220 \mathrm{MHz} \mathrm{GHz}$ and a FSR of $24.5 \mathrm{GHz}$, which is equivalent to a finesse of 111 based on measured magnitude responses of the lattice stages. Extinction ratio of better than $17 \mathrm{~dB}$ can be maintained across a total bandwidth of more than $1 \mathrm{THz}$, which allows an RF IBW of over $10 \mathrm{GHz}$ and a resolution bandwidth of better $250 \mathrm{MHz}$ for a channelizing receiver or spectrum analyzer.

The measured results serve as proof of concept that this design is a viable component in a multicasting RF channelizer system. Observed practical impediments such as excess insertion loss and degraded extinction ratio can be overcome in subsequent spins by adjusting the design parameters to better match those of fabricated devices. The fabricated filter has low SWaP, is thermally stable and relatively simple to tune.
The thermal crosstalk between rings may be calibrated out, or reduced by employing an isolation trench scheme to decrease the effective thermal conductivity between stages [6]. The
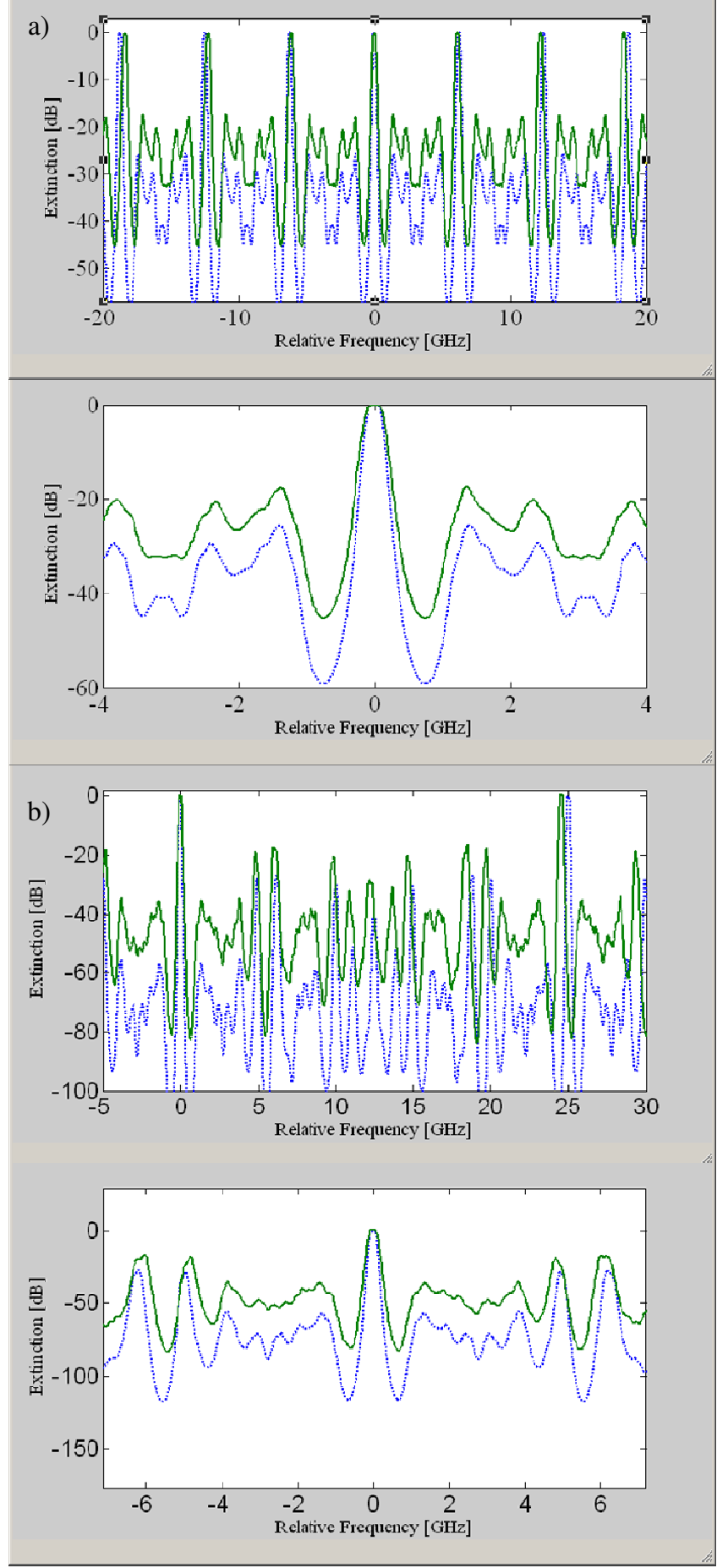

Fig. 4. Optimized magnitude response of compound filters calculated from measured magnitude response as compared to design (dotted lines). Curves are nomalized to peak transmision. a) nominal $6.25 \mathrm{GHz} 3$ stage filter, with 
measured FSR of $6.15 \mathrm{GHz}$ and $\sim 260 \mathrm{MHz}$ RBW. b) nominal $25 \mathrm{GHz} 6$ stage filter with measured FSR of $24.5 \mathrm{GHz}$ and $220 \mathrm{MHz} \mathrm{RBW}$.

overall optical bandwidth of this type of filter is observed to be greater than $1 \mathrm{THz}$. The upper bandwidth limitation for this type of design is determined by the magnitude of group velocity dispersion and polarization mode dispersion in the PIC platform used, and is likely to exceed that of other components (e.g. circulators, polarizers and demultiplexers) in a practical channelizer system. Improved finesse and passband characteristics may be achieved using this architecture as applied state of the art low loss waveguide PICs [7], allowing IBWs out to hundreds of gigahertz.

\section{ACKNOWLEDGMENTS}

The authors gratefully acknowledge support from the Office of Naval Research, Electronic Warfare Discovery and Invention program administered by Dr. Peter Craig, and SPAWAR Systems Center Pacific's In House Laboratory Independent Research program administered by Dr. Dave Rees. The authors also would like acknowledge A. Spector, A.
Fitting, J. Fujita, and J. Sun of Enablence USA for custom fabrication of PIC devices and helpful discussions.

\section{REFERENCES}

[1] B. Ortega et al., "Advanced optical processing of microwave signals", EURASIP J. on Applied Signal Processing 10 pp. 1462-1484, 2010.

[2] C.S. Bres et al., "Parametric photonic channelized RF receiver", Phot. Tech. Lett., 23(6) 344-346 (2011).

[3] V. Van, J. Lightwave Tech., "Synthesis of elliptic optical filters using mutually coupled microring resonators". vol. 25 no. 2 pp. 584-590, Feb. 2007.

[4] C.K. Madsen, "efficient architectures for exactly realizing optical filters with optimum bandpass designs," Photon. Technol. Lett., vol. 10, no. 8, pp. $1136-1138$.

[5] C.K. Madsen and J.H. Zhao, Optical Filter Design and Analysis: A Signal Processing Approach", New York: Wiley Interscience, 1999, pp 246-251

[6] P. Dong et al. "Thermally tunable silicon racetrack resonators with ultralow tuning power," Opt. Express 18(19),20298-20304 (2010).

[7] J.F. Bauters et al., "Planar waveguides with less than $0.1 \mathrm{~dB} / \mathrm{m}$ propagation loss fabricated with Wafer Bonding," Opt. Express 19(24),24090-24101 (2011). 